\title{
Visual prognostic value of the pattern electroretinogram in chiasmal compression
}

\author{
Dipak N Parmar, Ajit Sofat, Richard Bowman, John R Bartlett, Graham E Holder
}

\begin{abstract}
Backgroundlaims-The visual loss associated with compression of the optic chiasm by pituitary tumours may be transient or permanent, possibly related to the extent of irreversible retrograde degeneration to the retinal ganglion cells. The pattern electroretinogram (PERG) N95 component is thought to rise in relation to retinal ganglion cell function and hence may be a potential prognostic indicator for visual function following decompressive surgery.
\end{abstract}

Methods-The notes and electrodiagnostic records of 72 eyes from 36 patients with chiasmal compression were retrospectively analysed.

Results-The postoperative change in visual field was found to be associated with the PERG N95:P50 ratio $(p=0.01)$. Improvement in visual field was shown by a greater proportion of eyes with a normal N95:P50 ratio $(65 \%)$ than with an abnormal ratio $(27 \%)$. No change in visual field occurred in $26 \%$ of the eyes with a normal N95:P50 ratio compared with $67 \%$ of those with an abnormal ratio. Only $8 \%$ of eyes showed a worsening of visual field following surgery, in similar proportions for eyes with normal and abnormal N95:P50 ratios. There was no significant relationship with visual acuity.

Conclusion-The PERG is a useful visual prognostic indicator in the preoperative assessment of chiasmal compression.

(Br F Ophthalmol 2000;84:1024-1026)

Compression of the optic chiasm by tumours such as pituitary adenomas can lead to compromised visual function that may be transient, but may persist following decompressive surgery. The degree of postoperative recovery is thought to depend in part on the degree of structural damage involving retrograde degeneration to retinal ganglion cells. ${ }^{1-3}$ While the visual evoked potential (VEP) enables an assessment of chiasmal function, it provides no indication of possible retinal ganglion cell degeneration secondary to tumour compression, and hence is of no prognostic value with regard to postoperative visual outcome. ${ }^{4}$

The pattern electroretinogram (PERG) originates from the inner retinal layers, enabling an assessment of ganglion cell function, and is increasingly used in the assessment of anterior visual pathway dysfunction..$^{23-7}$ The normal PERG consists of a prominent positive component, P50, with a larger later negative component, N95. The positive P50 compo- nent is invariably affected in retinal and macular dysfunction, whereas the negative N95 component is principally affected in optic nerve disease. ${ }^{28-11}$ Furthermore, the ratio between N95 and P50 has been shown to be an effective measure of retinal ganglion cell function; in more severe ganglion cell dysfunction P50 may also show reduction.

Structural damage which compromises retinal ganglion cell function could therefore be expected to lead to PERG abnormalities associated with a poor postoperative visual outcome. The aim of the present study was to ascertain the potential of the PERG for predicting postoperative visual outcome in chiasmal compression.

\section{Methods}

We retrospectively analysed the electrodiagnostic records of 72 eyes from the notes of 36 patients, 23 women and 13 men, presenting to the Regional Neurosciences Unit, King's College Hospital, London. The median age was 55 years (range 26-81). Other than one patient with a suprasellar meningioma, all patients had pituitary adenomas. Surgical resection of tumour was performed using either a transphenoidal or transfrontal approach. Apart from compression of the optic pathway, there was no history of significant ophthalmological disease in any of the patients. Preoperative examination and electrophysiological studies including the PERG were carried out during the week before surgery. This included Snellen visual acuity and Goldmann visual fields, both of which were also recorded 3 months postoperatively.

The checkerboard transient PERG was recorded in accordance with the guidelines of the International Society for Clinical Electrophysiology of Vision (ISCEV). ${ }^{12}$ Gold foil recording electrodes (CH Electronics, London) were placed in the fornix of the lower eyelid and referred to ipsilateral outer canthus silver-silver chloride electrodes with a mid forehead earth. ${ }^{13}$ In early patients the signals were processed by a Nicolet CA-1000 averaging computer with internal artefact rejection and a bandwidth of $1-100 \mathrm{~Hz} / 3 \mathrm{~dB}$. For later patients a Neuroscience recording system was used with similar characteristics. There were a minimum of 200 averages per trial with a minimum of two trials performed to confirm the reproducibility of the waveform. Checkerboard reversal was performed by a Digitimer moving mirror stimulator subtending a total field of 14 degrees at the eye with an individual check size of 34 minutes. The mean luminance was $400 \mathrm{~cd} / \mathrm{m}^{2}$ with $89 \%$ contrast (non-linear, 
dropping to $200 \mathrm{~cd} / \mathrm{m}^{2}$ at the edges) and the stimulation rate for PERG was 4.8 reversals/s, with an analysis time of $200 \mathrm{~ms}$. The distance between the patient's eyes and the monitor was $60 \mathrm{~cm}$. The test was performed binocularly to facilitate fixation with undilated pupils and spectacle correction was used when appropriate. The effects of blink artefact were minimised by instructing the patient not to blink while the pattern was moving. Every 4-6 seconds stimulation was interrupted, the patient allowed to blink a few times, and stimulation resumed after signal stabilisation.

ANALYSIS OF DATA

The main outcome variables were the intraocular N95:P50 ratio obtained from the transient PERG, change in visual acuity, and visual field. The lower limit of the N95:P50 ratio was taken as 1.1. The degree of visual field defect was estimated as a quadrantanopia, hemianopia, or central scotoma. A score of 1 was given for each quadrant of visual field loss, as well as for a central scotoma, up to a maximum of 5 representing total loss of visual field. The extent of visual acuity loss was recorded by adding a score of 1 for each line of acuity lost after $6 / 5 \quad($ score $=0)$ to NPL (score=11). For statistical analysis Fisher's exact test (Stata Statistical Software, Release 5.0, Texas, USA, 1997) was used.

\section{Results}

Table 1 shows the postoperative change in visual field for eyes with normal and abnormal $(<1.1)$ preoperative N95:P50 ratios. These data provide strong evidence of an association between N95:P50 ratios and visual field change following surgery $(p=0.01)$. A greater proportion of eyes with a normal N95:P50 ratio $(64.91 \%)$ showed improvement in visual field than those with an abnormal ratio $(26.67 \%)$. Only $8.33 \%$ of eyes showed a worsening of visual field following surgery, in similar proportions for eyes with normal $(8.77 \%)$ and abnormal $(6.67 \%)$ N95:P50 ratios. The percentage of eyes whose visual fields remained unchanged was much greater in those with an abnormal N95:P50 ratio $(66.67 \%)$ than those with a normal N95:P50 ratio (26.32\%).

Table 2 shows the change in visual acuity postoperatively for eyes with normal and

Table 1 Change in visual field $(V F)$ for eyes with normal and abnormal $(<1.1)$ preoperative N95:P50 ratios. Column percentages are shown.

\begin{tabular}{lccc}
\hline$V F$ & Abnormal N95:P50 & Normal N95:P50 & Total \\
\hline Worse & $1(6.67 \%)$ & $5(8.77 \%)$ & $6(8.33 \%)$ \\
No change & $10(66.67 \%)$ & $15(26.32 \%)$ & $25(34.72 \%)$ \\
Improved & $4(26.67 \%)$ & $37(64.91 \%)$ & $41(56.94 \%)$ \\
Total & $15(100.00 \%)$ & $57(100.00 \%)$ & $72(100.00 \%)$ \\
\hline
\end{tabular}

Fisher's exact test $(\mathrm{p}=0.01)$

Table 2 Change in visual acuity (VA) for eyes with normal and abnormal $(<1.1)$ preoperative N95:P50 ratios. Column percentages are shown

\begin{tabular}{lcll}
\hline VA & Abnormal N95:P50 & Normal N95:P50 & Total \\
\hline Worse & $4(26.67 \%)$ & $13(22.81 \%)$ & $17(23.61 \%)$ \\
No change & $2(13.33 \%)$ & $21(36.84 \%)$ & $23(31.94 \%)$ \\
Improved & $9(60.00 \%)$ & $23(40.35 \%)$ & $32(44.44 \%)$ \\
Total & $15(100.00 \%)$ & $57(100.00 \%)$ & $72(100.00 \%)$ \\
\hline
\end{tabular}

Fisher's exact test $(\mathrm{p}=0.224)$. abnormal (<1.1) preoperative N95:P50 ratios. A slightly lower proportion of eyes with a normal N95:P50 ratio lost visual acuity postoperatively $(22.81 \%)$ compared with those with an abnormal ratio (26.67\%). Improvement in visual acuity was shown by $40.35 \%$ of eyes with a normal N95:P50 ratio compared with $60.00 \%$ of those with an abnormal ratio. These differences were not statistically significant.

\section{Discussion}

The improvement in visual function following surgical decompression of the optic nerve and chiasm for pituitary tumours is highly variable, with the degree of retrograde degeneration to the retinal ganglion cells considered an important determinant of final visual outcome. ${ }^{2} 3714$ Compression of the optic nerve or chiasm may lead to initial demyelination without significant axonopathy. ${ }^{15}$ However, following prolonged compression, retrograde degeneration of ganglion cells may occur, compromising the potential visual improvement following surgical decompression. ${ }^{237}$ Previous studies involving compressive lesions of the optic nerve have suggested that an abnormal preoperative PERG implies a poor visual result following decompressive surgery. ${ }^{71516}$ The present larger study confirms and extends those observations.

The results demonstrate that the chances of visual field improvement following surgery are greater in those eyes with a normal PERG. The proportion of eyes with a normal preoperative PERG that worsened in visual field following surgery was low $(8.77 \%)$. This may be related to surgical trauma or the impact of surgery on the vasculature of the optic nerve and chiasm causing relative ischaemia. A larger proportion of eyes with a normal PERG showed no change in visual field $(26.32 \%)$. A possible temporal latency between structural compression of the optic chiasm and attenuation of the PERG may explain this. Conversely, a similar proportion of eyes with abnormal PERG showed an improvement in visual field $(26.67 \%)$. A possible explanation is that, although retrograde degeneration had already occurred, additional transient reversible compressive effects at the chiasm were also present. In contrast, the lack of statistical significance with regard to visual acuity is surprising, given that the retinal contribution to the PERG is largely central. ${ }^{2}$ While this may be caused by the relatively small number of patients in our study, it is possible that chiasmal tumours may cause compression of peripheral macular nerve fibres first, before affecting central macular fibres, and thus visual acuity, at a later stage.

As a putative prognostic measure, the PERG is more appropriate than the VEP. The profound effects which may be present in the pattern VEP enable an assessment of chiasmal function but reflect only the primary effects of the compressive lesion. ${ }^{4}$ The VEP may thus normalise following surgery, or remain abnormal. Importantly, the VEP provides no indication of possible retinal ganglion cell degeneration secondary to tumour compression, and hence is of no prognostic value with regard to postoperative visual outcome. In contrast, 
abnormalities of the PERG reflect dysfunction at a more distal site, specifically the retinal ganglion cells, and enable an assessment of visual prognosis.

In conclusion, the results confirm that the PERG may be a useful prognostic indicator in the preoperative assessment of chiasmal compression. An eye with demonstrable retinal ganglion cell dysfunction, as shown by an abnormal N95:P50 ratio, is less likely to be associated with clinical improvement following surgery than an eye with a normal PERG. A prospective study may assist in the identification of the exact parameters to provide a more accurate prognosis in an individual patient.

1 Elkington S. Pituitary adenoma. Preoperative symptomatology in a series of 260 patients. $B r f$ Ophthalmol 1968;52:322-8

2 Holder GE. The pattern electroretinogram in anterior visual pathway dysfunction and its relationship to the pattern visual evoked potential: a personal clinical review of 743 eyes. Eye 1997;11:924-34.

3 Holder GE. Significance of abnormal pattern electroretinography in anterior visual pathway dysfunction. $\mathrm{Br} \mathcal{F} \mathrm{Oph}$ ography in anterior visual
thalmol 1987;71:166-71.

4 Brecelj J. A VEP study of the visual pathway function in compressive lesions of the optic chiasm. Full-field versus compressive lesions of the optic chiasm. Full-field versus
half-field stimulation. Electroencephalogr Clin Neurophysiol half-field stimula

1992;84:209-18.
Maffei L, Fiorentini A, Bisti S, et al. Pattern ERG in the monkey after section of the optic nerve. Exp Brain Res 1985;59:423-5.
6 Fiorentini A, Maffei L, Pirchio M, et al. The ERG in response to alternating gratings in patients with diseases of the peripheral visual pathway. Invest Ophthalmol Vis Sci 1981;21:490-3.

7 Ruther K, Ehlich P, Philipp A, et al. Prognostic value of the pattern electroretinogram in cases of tumors affecting the optic pathway. Graefe's Arch Clin Exp Ophthalmol 1998;236: 259-63.

8 Berninger TA, Arden GB. The pattern electroretinogram. Eye 1988;2(Suppl):S257-83.

9 Ryan S, Arden GB. Electrophysiological discrimination between retinal and optic nerve disorders. Doc Ophthalmol 1988;68:247-55.

10 Holder GE. Pattern electroretinography in patients with delayed pattern visual evoked potentials due to distal anterior visual pathway dysfunction. I Neurol Neurosurg Psychiatry 1989;52:1364-8.

11 Berninger TA, Schuurmans RP. Spatial tuning of the pattern ERG across temporal frequency. Doc Ophthalmol 1985;61:17-25.

12 Marmor MF, Holder GE, Porciatti V, et al. Guidelines for basic pattern electroretinography. Recommendations by the International Society for Clinical Electrophysiology of Vision. Doc Ophthalmol 1996;91:291-8.

13 Arden GB, Carter RM, Hogg C, et al. A gold foil electrode: extending the horizons for clinical electroretinography. Invest Ophthalmol Vis Sci 1979;18:421-6.

14 Kayan A, Earl C. Compressive lesions of the optic nerve and chiasm. Pattern of recovery of vision following surgical treatment. Brain 1975;98:13-28.

15 Kaufmann D, Wray SH, Lorance R, et al. An analysis of the pathophysiology and the development of treatment strategies for compressive optic nerve lesions using pattern electroretinogram and visual evoked potential. Neurology 1986;36(Suppl 1):232.

16 Sadowski B, Altenmuller E, Zrenner E. Electrophysiological and psychophysical examination in patients with optic nerve compression. Neuroophthalmology 1995;15:223-31. 